\title{
Programa nutricional, conductual y de actividad física para el abordaje de la obesidad tipo 1 y 2
}

\author{
Nutritional, behavioral and physical activity program \\ to address type 1 and 2 obesity
}

\begin{abstract}
Fredy Alexander Rodríguez
Castellanos $^{* *}$

ORCID 0000-0002-9086-5598

Ángela Yazmín Gálvez Pardo ORCID 0000-0002-8041-4646

Edward Giovanni Ayala Noy ORCID 0000-0001-5366-5055

Nelsy Lorena Fonseca Torres ORCID 0000-0003-4276-612X

Universidad Santo Tomás, Colombia

Diana Milena García Beltrán ORCID 0000-0003-4180-7065

Fundación Universitaria Konrad Lorenz
\end{abstract}

Recibido: 12 de noviembre de 2017 Revisado: 28 de diciembre de 2017 Aceptado: 29 de enero de 2018

\section{Resumen}

La obesidad es un factor de riesgo para muchas enfermedades crónicas. Se ha propuesto que debe ser contrarrestada con hábitos nutricionales y de actividad física saludables. En un diseño de línea de base múltiple entre participantes, la presente investigación tuvo como propósito identificar cambios en el número de pasos, gasto calórico, aptitud física y composición corporal en 4 adultos, mediante una intervención multicomponente (es decir, entrenamiento concurrente, dieta, educación nutricional y coaching conductual) durante 7 semanas. El aumento porcentual en el número de pasos fue de $23.93 \%$ y del gasto kilocalórico de $9.25 \%$ respecto a la línea de base, relacionándose principalmente con una disminución del perímetro de cintura y un aumento en la flexibilidad en todos los participantes; es probable que las diferencias en los resultados se expliquen principalmente por adherencia. Finalmente, se abordan aspectos a mejorar para futuras investigaciones.

Palabras clave: Obesidad, actividad física, nutrición, ejercicio, adherencia.

Artículo de Investigación. Este trabajo es producto del proyecto de investigación 2016, con financiación FODEIN, Proyecto titulado "Evaluación de la Efectividad de un Programa Conductual para Controlar el Peso con y sin Estrategias de Aceptación y Compromiso". http://dx.doi. org/10.15332/s1794-9998.2018.0001.05

Fredy Alexander Rodríguez Castellanos. Universidad Santo Tomás. Colombia. Dirección postal: Kilómetro 1,6, Cl. 209, Bogotá, Colombia. Correo electrónico: fredyrodriguez@usantotomas.edu.co 


\section{Abstract}

Obesity is a risk factor for many chronic diseases which must be counteracted with healthy physical activity and better nutritional habits. Using a multiple baseline design among participants, we aimed to identify changes in: number of steps, caloric expenditure, physical fitness and body composition in 4 adults. The intervention consisted of concurrent training, diet, nutritional education and behavioral coaching. After the intervention, the number of steps increased by $23.93 \%$ and kilocaloric rose by $9.25 \%$ related to baseline. These changes were associated with a decrease in waist circumference and increase in flexibility in all participants; It's possible that the differences in the results are explained mainly by adherence. Finally, aspects to be improved are addressed for future research.

Keywords: Obesity, physical activity, nutrition, exercise, adherence.

\section{Introducción}

La obesidad es un problema de salud pública a nivel mundial; por ejemplo, en China la cifra de obesidad llegó a los 15 millones de personas entre 2010 y 2014, siendo mayor la prevalencia en áreas rurales y entre las edades de 15 a 49 años (He et al., 2017). China se encuentra cada vez más cerca de desplazar a Estados Unidos del primer lugar (Foncillas, 2016). Parecería ser un problema que afecta principalmente a las naciones desarrolladas pero que se ha visto también en aumento en los países en vía de desarrollo (Ministerio de Protección Social, 2005).

En Colombia, al igual que en China y los demás países, la mayor prevalencia en los adultos de 18 a 64 años se evidencia en el sobrepeso con 37,7 puntos porcentuales, siendo preocupante también la cifra de un $18 \%$ para la obesidad; ahora bien, al comparar los resultados de la Encuesta Nacional de la Situación Nutricional del 2010 con la del 2015 se presenta un incremento de 2,2\% para la obesidad y de 3,1\% para el sobrepeso (Ministerio de Salud y Protección Social, Instituto Colombiano de Bienestar Familiar, Instituto Nacional de Salud \& Universidad Nacional, 2015). A su vez, la obesidad es un factor de riesgo para la diabetes, enfermedades cardiovasculares, osteoartritis y daños al sistema inmunológico, las dos primeras con cifras destacadas para hombres y mujeres respecto a morbimortalidad en el país (Ministerio de Salud y Protección Social, 2016).

El índice de masa corporal (IMC) es la medida utilizada por excelencia para identificar posibles problemas de desnutrición, sobrepeso y obesidad en la población adulta mediante la relación de peso y estatura. El rango entre 30 y 34.9 equivale a obesidad tipo 1, mientras que un IMC entre $35 \mathrm{y}$ 39.9 corresponde a obesidad tipo 2 .

Aunque se han establecido algunas influencias genéticas sobre los comportamientos de riesgo para el desarrollo de la obesidad como son la ingesta excesiva de alimentos y la insuficiente actividad física, se considera primordialmente un asunto de influencia ambiental con alguna asociación con la depresión (Hernández \& Blazer, 2006), lo que hace pertinente la intervención psicológica además de la nutricional y de actividad física.

La obesidad es una enfermedad compleja que requiere una intervención multicomponente y multisectorial (Dietz, Lee, Wechsler, Malepati \& Sherry, 2007), pues de acuerdo a evidencias científicas. Las pérdidas de peso suelen ser mayores cuando se realizan intervenciones durante un período de tiempo prolongado (dos años); sin embargo, puede existir una recuperación de peso en un $20 \%$ de los casos entre los seis meses al año posterior a la intervención (Sacks et al., 2009). 
Debido a esta problemática, en Colombia se han creado políticas públicas para promover el ejercicio y la alimentación saludable como la ley 1355 de 2009, pese a esto, el mayor reto es lograr adherencia al ejercicio (Burgess, Hassmén \& Pumpa, 2017). Los programas de intervención son eficaces para el tratamiento de la obesidad.Sin embargo, la asistencia de los usuarios tiende a ser irregular, por tal razón se deben crear estrategias que motiven a los participantes a realizar ejercicio o actividad física y llevar una dieta sana en consonancia con sus gustos e intereses.

En cuanto a la realización del ejercicio físico, existen varias propuestas para diseñar un programa de entrenamiento efectivo para la obesidad, entre las que sobresale el entrenamiento concurrente cuando los participantes tienen un estatus bajo de aptitud física y no tienen mucho tiempo libre (Monteiro et al., 2015). De acuerdo con Peña, Heredia, Aguilera, Da Silva y Del Rosso (2016), el entrenamiento concurrente consiste en la combinación del entrenamiento de la fuerza y la resistencia en la misma sesión, esto con el fin de estimular la mayor cantidad de adaptaciones en un mínimo de tiempo lo cual hace que las cargas programadas sean altamente especializadas.

Sin embargo, como se mencionó antes, es necesario contar con los intereses y gustos de los participantes para garantizar la adherencia a un programa de ejercicio, pues la motivación es uno de los elementos clave para conseguir el hábito (Molinero, Salguero \& Márquez, 2011). Es decir, no todas las personas gustarán de pertenecer a un programa de ejercicio, así que es imperativo ofrecer alternativas para el incremento de la actividad física, reflejada en el aumento de pasos diarios y el incremento del gasto calórico.

Las personas adultas deberían realizar como mínimo 10.000 pasos diarios para tener algún beneficio para la salud (Tudor-Locke et al., 2011). La Organización Mundial de la Salud (OMS, 2017), también recomienda un mínimo de 150 minutos semanales de actividad física moderada o 75 minutos de actividad intensa en 3 ocasiones semanales; si a lo anterior se le suma una adecuada dieta, entonces podría buscarse con mayor probabilidad de éxito el balance calórico para enfrentar la obesidad.

Por su parte, la elección de una dieta también debe dirigirse a la preferencia del individuo buscando estrategias que permitan el cambio de hábitos a largo plazo con patrones de alimentación que sean prácticos, alcanzables y sostenibles (Dietz et al., 2015). Una dieta de pérdida de peso puede tener diferentes variaciones en la composición de macronutrientes (baja en carbohidratos, baja en grasa, dieta mediterránea, con baja carga glicémica, entre otras). Sin embargo, las dietas con restricción calórica, en lugar de la modificación de macronutrientes, son una clave para la pérdida de peso. Ahora bien, se considera exitosa aquella dieta restrictiva que pueda adherir a la persona durante varios meses con pérdidas de peso entre el $5 \%$ al $10 \%$, teniendo en cuenta que mayores pérdidas de peso están relacionadas con la disminución de factores de riesgo para múltiples enfermedades (Wadden, Webb, Moran, \& Brooke, 2012). Además de la dieta, es necesario realizar una adecuada educación nutricional acerca de funciones de macronutrientes e intercambios de la dieta y porciones.

Una dieta hipocalórica, junto a un gasto calórico medido con acelerómetros podrían hacer la diferencia, puesto que existen investigaciones relacionadas con el uso de la tecnología y componentes conductuales que favorecen moderadamente el incremento del gasto calórico en personas con sobrepeso y obesidad (Donaldson \& Normand, 2009). Los componentes conductuales de establecimiento de objetivos, retroalimentación y automonitoreo, se han utilizado en diversos contextos y temáticas (Bach \& McCracken, s.f.), y los tres pueden utilizarse en la adopción de ambos hábitos saludables e incluso se encuentran inmersos en los acelerómetros comerciales, los cuales han obtenido resultados satisfactorios en validez y confiabilidad (Kang \& Rowe, 2015).

Éstos instrumentos (acelerómetros) pueden medir el número de pasos realizados y el gasto calórico diario, así como los usuarios pueden obtener un cálculo de las calorías ingeridas al seleccionar una lista de alimentos, gracias a una aplicación anexa 
al dispositivo. De esta forma, las personas pueden a través de un coaching conductual ser asesoradas y retroalimentadas semanalmente por otro individuo sobre las metas establecidas, monitoreando constantemente su acercamiento a las mismas, tal como lo expone Valbuena (2013).

Además del número de pasos y las calorías gastadas, se deben tener otras medidas para identificar cambios a nivel corporal, como consecuencia de la intervención, y son las resultantes de la evaluación de la aptitud física, considerada como la capacidad que posee un individuo para desarrollar sus actividades cotidianas sin quedar exhausto; se compone de cuatro aspectos: a) resistencia cardiorrespiratoria, b) aptitud musculo esquelética, c) flexibilidad y d) peso y composición corporal (Heyward, 2008).

La resistencia cardiorrespiratoria es la capacidad conjunta de éstos dos sistemas para aportar el oxígeno y las sustancias nutritivas a los músculos y puede medirse con la prueba de Caminata por 6 Minutos (Butland, Pang, Gross, Woodcock \& Geddes, 1982). Esta prueba fue desarrollada para ser aplicada en pacientes con enfermedad respiratoria crónica, pero es conveniente usarla con personas en condición de obesidad (Larsson \& Reynisdottir, 2008).

La aptitud músculo esquelética es la capacidad conjunta de estos dos sistemas para realizar una acción; puede medirse con el Test de Fuerza Prensil (Jakobsen, Rask \& Kondrup, 2010) y el Test de Sentarse y Levantarse de la Silla (Millor, Lecumberri, Gómez, Martínez-Ramírez \& Izquierdo, 2013).

La flexibilidad es la capacidad de movilizar con fluidez y en toda su amplitud las articulaciones, es posible evaluarlo con el Test de Seat and Reach (Wells y Dillon, 1952).

El peso es una medida insuficiente pues no discrimina grasa, hueso, músculo y demás tejido, así que se requiere medir variables de la composición corporal como el porcentaje graso y la grasa visceral, en este caso mediante una báscula de bioimpedancia. Además de lo anterior es necesario medir la talla y el perímetro de la cintura.
La presente investigación de intervención multidisciplinar se constituye en el primer producto de un proyecto piloto con 4 participantes que incluye además de las intervenciones planteadas, un entrenamiento en aceptación y compromiso en la siguiente fase, para fortalecer los hábitos saludables de alimentación y actividad física.

El objetivo general es identificar si una intervención nutricional (dieta y educación nutricional), de actividad física (entrenamiento concurrente y actividad física en el tiempo libre), y coaching conductual (establecimiento de metas, automonitoreo y retroalimentación de pasos y gasto calórico) tiene un efecto benéfico sobre la composición corporal y la aptitud física en dos hombres clasificados con obesidad tipo 1 y dos mujeres clasificadas con obesidad tipo 2 .

\section{Método}

\section{Diseño}

Línea de base múltiple entre participantes (Barlow, Nock \& Hersen, 2009), sin reversión, para evaluar la efectividad de la intervención de tipo multicomponente (Kazdin, 2000) en 4 participantes, el cual permite identificar tendencias en la variable dependiente, sin desconocer las particularidades de cada individuo entre la línea base y la intervención.

\section{Participantes}

Cuatro adultos voluntarios entre los 32 y 50 años de edad, funcionarios de una entidad estatal con trabajos administrativos; dos hombres con obesidad tipo 1 (participantes 1 y 4 ) y dos mujeres con obesidad tipo 2 (participantes 2 y 3), de acuerdo al IMC. Los criterios de exclusión fueron haber participado un mes atrás de un programa estructurado de ejercicio o deporte, con intolerancias alimenticias, un diagnóstico previo de bulimia/ anorexia, tratamiento con corticoides, antihistamínicos, antidepresivos y contraceptivos orales al momento de la selección; uno de los hombres reporta problemas cardiovasculares. 
Como criterio de inclusión debían tener un nivel de aptitud física en VO2 entre medio y bajo en el Cuestionario de Medición de la Capacidad Física sin Hacer Ejercicio; el participante 2 tuvo un nivel bajo y los demás tuvieron uno medio. Además, en el cuestionario de Frecuencia de Consumo de Alimentos debería cumplir con una de las siguientes opciones: alta ingesta de grasas o baja de frutas y vegetales.

\section{Instrumentos}

La podometría y el cálculo del gasto calórico diario se obtuvo con 4 monitores Fitbit ${ }^{\circledR}$ Alta $^{T M}$ los cuales poseen acelerómetros de última generación. En particular sobre esta marca se han hecho estudios de acuerdo entre observadores (Valbuena, 2013) con un porcentaje de acuerdo entre el instrumento y el observador superior al $99 \%$.

El peso y la composición corporal (porcentaje graso y porcentaje de grasa visceral) se obtuvieron con la ayuda de una báscula de bioimpedancia Tanita Iron Man BC-554 (precisión 50 g a 100 g), la estatura se midió con un tallimetro Holtain $(0$ - $209 \mathrm{~cm}$; precisión de 0,1 cm) y el perímetro de la cintura se valoró con una cinta métrica (rango de medición 0-150 cm; precisión de $1 \mathrm{~mm}$ ). Para cada una de las valoraciones se tuvo en cuenta el protocolo de la Sociedad Internacional para el Avance de la Cineantropometría.

A modo de seguimiento de la adopción de la dieta alimentaria los investigadores utilizaron la técnica de Recordatorio de 24 Horas, durante 5 días lo que permitió recolectar datos sobre la ingesta reciente. Mediante el uso de una aplicación de chat los participantes enviaron fotos sobre lo que consumían, lo que permitió disminuir los sesgos (Ferrari, 2013).

A continuación se para la evaluación de la aptitud física seleccionaron instrumentos que midieran sus componentes: fuerza de tren superior e inferior, resistencia cardiovascular y flexibilidad:

El Test de Fuerza Prensil (Jakobsen et al., 2010), utilizado para medir la fuerza general de miembro superior, dado que a medida que aumenta la edad, ésta tiende a disminuir en hombres y mujeres. Estos investigadores encontraron una correlación inversa entre la fuerza prensil y movilidad en sujetos sanos $(r=-0,31, p=0,002)$ y también en personas hospitalizadas $(r=-0,59, p<0,001)$; dicho test se utiliza como punto de partida para la preservación del fitness muscular como indicador de salud cardiovascular (Ramírez-Vélez, MenesesEchavez, González-Ruiz \& Correa, 2014).

El Test de Sentarse y Levantarse de la Silla, sirve para evaluar la potencia de miembros inferiores al levantarse y sentarse de una silla en 30 segundos en adultos; según Cobo-Mejia et al. (2016), el alfa de Cronbach fue de 0,708, mientras la reproducibilidad de las pruebas obtuvo un coeficiente de confiabilidad interevaluador entre 0,851 y 0,960 con un intervalo de confianza del $95 \%$. El instrumento fue tomado de la batería de Senior Fitness Test diseñada por Rikli y Jones (2001).

La prueba de los Seis Minutos Caminando se realiza para detectar la distancia que puede ser recorrida con paso rápido, sobre una superficie plana y dura durante seis minutos (American Thoracic Society Statement, 2002). Es una prueba confiable y válida para detectar el estatus de aptitud cardiorrespiratoria (Segura \& Martínez, 2009).

Por su parte el Test de Sit and Reach es usado para medir la flexibilidad; de acuerdo con Ayala, Sainz de Baranda, Ste Croix y Santonja (2012), ha demostrado poseer una elevada fiabilidad, con valores que oscilan entre 0,89 y 0,99 , independientemente del sexo y del protocolo utilizado. Se puede realizar con un cajón de Wells, en posición sedente, descalzo y sin flexión de rodillas, para el caso de mujeres se utilizó la versión modificada de Hoeger y Hopkins (1992).

El Cuestionario de Medición de la Capacidad Física sin Hacer Ejercicio, evita la utilización de pruebas de ejercicio; Jackson et al. (1990) identificaron un adecuado valor de predicción de ésta variable con el estadístico de Regresión Lineal Múltiple, respecto a pliegues cutáneos e IMC con un $\mathrm{R} \geq 0,78$ de manera inversamente proporcional.

El Cuestionario de Frecuencia de Consumo de Alimentos (Block, Gillespie, Rosenbaum \& Jenson, 
2000), ha sido validado con el instrumento Block 100 -items Food Frequency Questionnaire, el cual es considerado como el "gold estándar", para esto se aplicó la correlación de Spearman obteniendo un nivel de predicción bueno $(r>0,60)$ con respecto a la ingesta dietaria de grasas y una excelente correlación con las porciones de frutas y verduras $(r=0,71)$.

\section{Procedimiento}

Identificada una institución gubernamental con un centro deportivo dotado de gimnasio, se procedió a entrevistar los interesados en el proyecto, además se obtuvo su IMC y se aplicó el Cuestionario de Capacidad Física Sin Hacer Ejercicio y el Cuestionario de Frecuencia de Consumo de Alimentos con el fin de realizar la selección.

Una vez obtenida la aprobación de la institución y los consentimientos informados, los investigadores entregaron los monitores inalámbricos con la pantalla oculta para los participantes el primer día; al final de cada semana de la línea de base eran recolectados los datos de pasos y kilocalorías (Kcal) gastadas mediante bluetooth, enviándolos a la cuenta creada para cada monitor en la plataforma Fitbit $®$.

También en la primera semana de la línea de base, se realizaron las evaluaciones de aptitud física, composición corporal y la historia dietaría, ésta última mediante entrevista.

Posteriormente, la primera semana de intervención inició con la retroalimentación del promedio diario de los pasos y las Kcal gastadas diariamente durante la línea de base, generándose metas al respecto con base en la guía de Coaching conductual de Valbuena (2013).

En el mismo periodo, a los participantes 1 y 2 se les entregó una dieta con una restricción calórica de $800 \mathrm{Kcal}$, mientras que los participantes $3 \mathrm{y}$ 4 tuvieron una restricción de $500 \mathrm{Kcal}$; la distribución en macronutrientes para todos los partici- pantes fue de un $55 \%$ carbohidratos, $20 \%$ proteína y $25 \%$ grasa con un consumo de ocho porciones de frutas y tres porciones de verduras durante el día. Adicional a lo anterior, se realizó educación nutricional en una sesión de 2 horas acerca de las leyes de la alimentación (completa, equilibrada, suficiente, adecuada e inocua), la importancia de cada nutriente en la dieta y el consumo fraccionado de alimentos durante el día manejando porciones más pequeñas.

Desde el inicio de la intervención se encontraban disponibles los planes de entrenamiento concurrente en el gimnasio con la rutina para cada semana, con un entrenador dispuesto a apoyar a los participantes en el desarrollo de su programa que constaba de mínimo 2 sesiones semanales. Las dos primeras semanas fue solo entrenamiento de aprendizaje y adaptación para evitar lesiones.

En la segunda semana de intervención se afianzó el proceso nutricional mediante la estimación de la ingesta con un recordatorio 24 horas 5 días, lo que permitió conocer lo aplicado en la educación nutricional. Asimismo, al final de cada semana un investigador retroalimentaba y establecía con el participante las metas de Kcal y pasos para la siguiente, con base en el promedio de esa misma semana.

La última semana de intervención se realizaron nuevamente las pruebas de aptitud física y composición corporal.

\section{Resultados}

Los participantes de la investigación tenían una edad promedio de $38 \pm 8,4$ años y una talla de $165,8 \pm 6,5 \mathrm{cms}$. Todos ellos aumentaron los pasos y el gasto energético respecto a la línea de base; comparando el promedio de pasos diarios entre línea base e intervención, el participante 1 tuvo el menor aumento con $11,78 \%$, el participante 2 fue el de mayor con $44,09 \%$, el participante 3 tuvo $23,94 \%$ de diferencia y el participante 4 de $15,91 \%$ (figura 1). 
Figura 1.

Promedio de pasos diarios realizados por semana de cada participante.

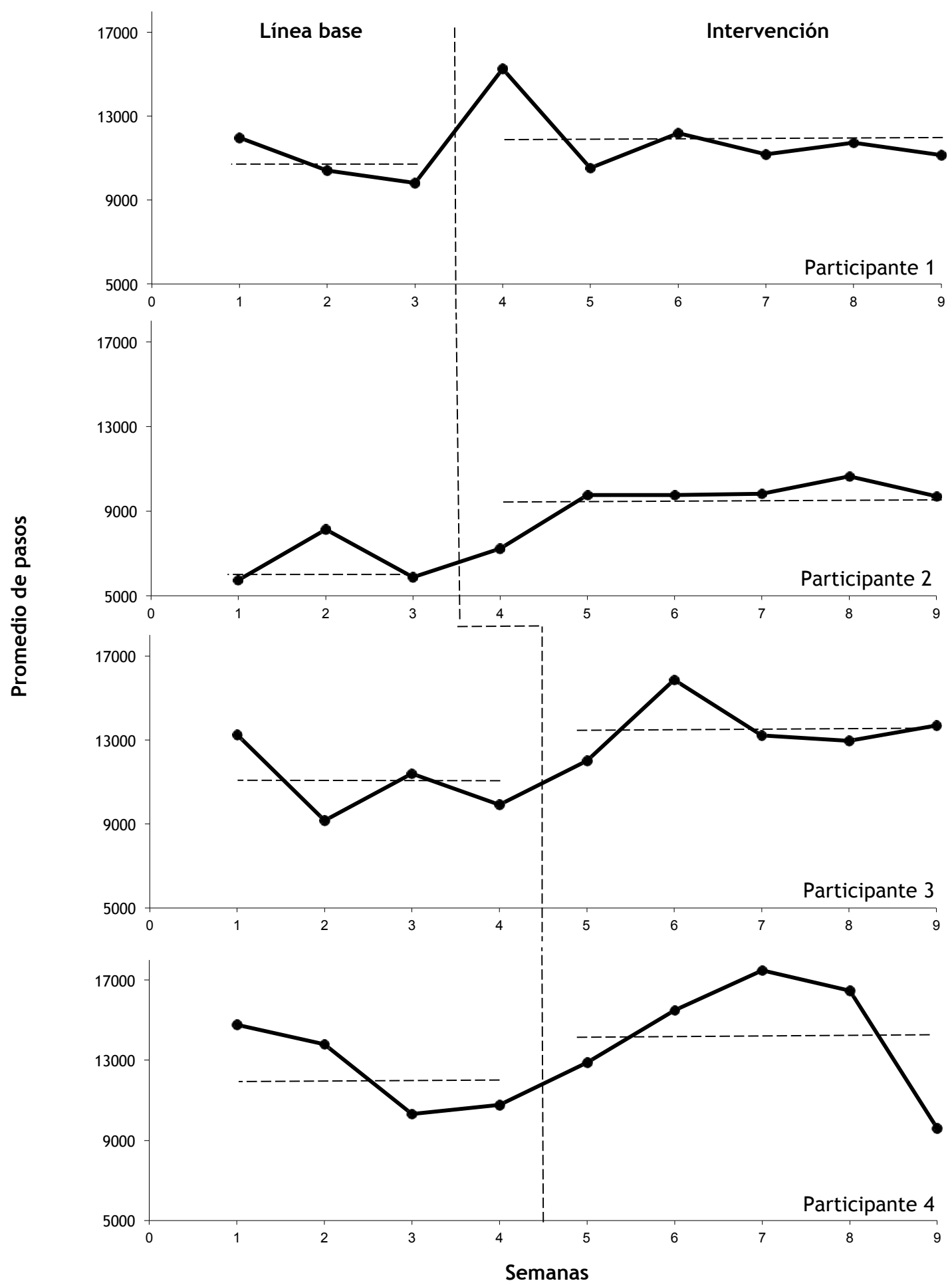

Fuente: Elaboración propia 
De forma proporcional, se aumentó el promedio de gasto en Kcal diario en la segunda fase; el mayor aumento fue del participante 2 con $18,83 \%$, seguido del participante 3 con $8,52 \%$, el participante 4 con $6,82 \%$ y finalmente el participante 1 con 2,86\% (figura 2).

Figura 2.

Promedio gasto kilocalórico realizado por semana de cada participante.

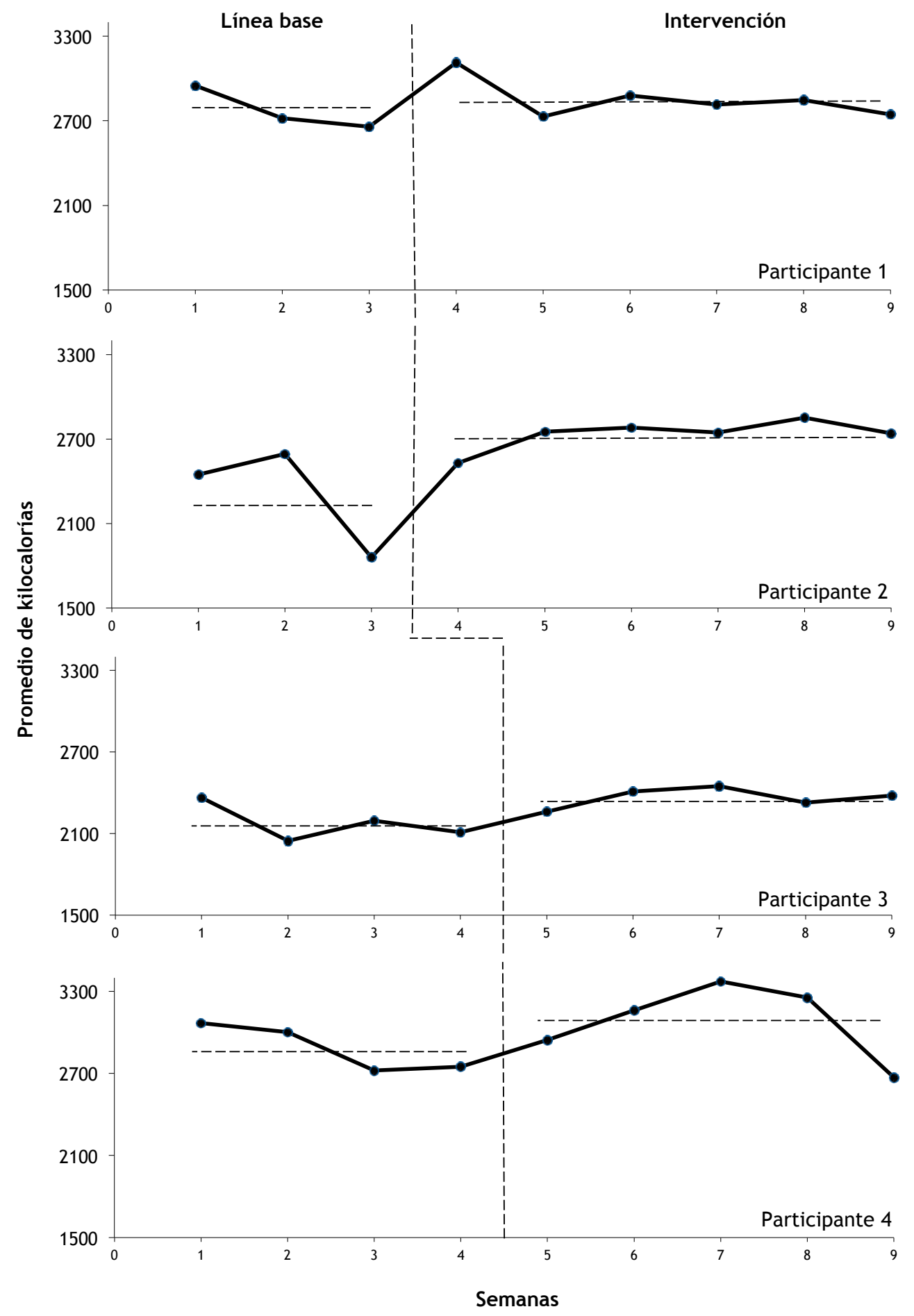

Fuente: Elaboración propia 
Ambas figuras muestran una tendencia decreciente a medida que avanza la primera fase y también se identifica la similitud en la segunda fase para cada participante.

Luego de la intervención hubo una disminución en el peso; las pérdidas oscilaron entre $3,3 \mathrm{~kg}$ a 0,9 kg con excepción del participante 2; sin embargo, en la totalidad se redujo el perímetro de cintura, siendo el participante 4 el de mayor reducción en ambos aspectos.
Respecto al porcentaje graso, los resultados muestran un incremento entre el $0,5 \%$ y el $3,1 \%$. Solamente el participante 1 disminuyó el porcentaje graso y el de grasa visceral, mientras que el participante 3 aumento en ambos aspectos. Los participantes 1,3 y 4 presentaron una reducción en el IMC entre 0,34 a 1,1 kg/m2, el participante 2 mantuvo el IMC, al igual que el peso (tabla 1).

Tabla 1.

Datos antropométricos pre-intervención y pos-intervención tradicional de cada participante.

\begin{tabular}{|c|c|c|c|c|c|c|c|c|c|c|c|c|c|c|c|c|}
\hline \multirow{2}{*}{ 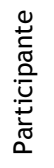 } & \multirow{2}{*}{$\begin{array}{l}\text { Talla } \\
\text { (cms.) }\end{array}$} & \multicolumn{3}{|c|}{ Peso Corporal (kgs.) } & \multicolumn{3}{|c|}{$\begin{array}{l}\text { Índice de Masa } \\
\text { Corporal (IMC) }\end{array}$} & \multicolumn{3}{|c|}{$\begin{array}{l}\text { Perímetro de cintura } \\
\text { (cms.) }\end{array}$} & \multicolumn{3}{|c|}{ Porcentaje graso (\%) } & \multicolumn{3}{|c|}{ Grasa visceral (\%) } \\
\hline & & Inicial & Final & Cambio & Inicial & Final & Cambio & Inicial & Final & Cambio & Inicial & Final & Cambio & Inicial & Final & Cambio \\
\hline 1 & 170.2 & 93.5 & 92.5 & -1 & 32.27 & 31.93 & -0.34 & 107.8 & 106.5 & -1.3 & 31.7 & 29 & -2.7 & 16 & 14 & -2 \\
\hline 2 & 161.2 & 104 & 104 & 0 & 40.02 & 40.02 & 0 & 100.3 & 96.2 & -4.1 & 48.5 & 49.1 & 0.6 & 12 & 12 & 0 \\
\hline 3 & 159.3 & 70.2 & 69.3 & -0.9 & 27.66 & 27.3 & -0.35 & 85.9 & 83.9 & -2 & 35 & 38.1 & 3.1 & 5 & 6 & 1 \\
\hline 4 & 172.5 & 91.2 & 87.9 & -3.3 & 30.64 & 29.54 & -1.1 & 99.6 & 94.4 & -5.2 & 24.6 & 25.1 & 0.5 & 10 & 10 & 0 \\
\hline
\end{tabular}

Respecto a la aptitud física de los participantes, todos aumentaron su flexibilidad y fuerza prensil, solamente los dos primeros participantes incrementaron su capacidad cardiorrespiratoria, entre tanto, el participante 2 fue quien mejor desem- peño presentó en el test de los 6 minutos caminando. Los participantes 3 y 4 no mejoraron sus puntajes en Squat 30", ni en el test de 6 minutos caminando (tabla 2).

Tabla 2.

Pruebas físicas pre-intervención y pos-intervención tradicional de cada participante.

\begin{tabular}{|c|c|c|c|c|c|c|c|c|c|c|c|c|c|}
\hline \multirow{2}{*}{ 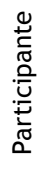 } & \multirow{2}{*}{$\begin{array}{l}\text { Talla } \\
\text { (cms.) }\end{array}$} & \multicolumn{3}{|c|}{ Set and reach $(\mathrm{cm})$} & \multicolumn{3}{|c|}{6 min. caminando (mts) } & \multicolumn{3}{|c|}{ Fuerza prensil (Newton) } & \multicolumn{3}{|c|}{ Squat 30" (repetición) } \\
\hline & & Inicial & Final & Cambio & Inicial & Final & Cambio & Inicial & Final & Cambio & Inicial & Final & Cambio \\
\hline 1 & 170.2 & -27.5 & -11 & 16.5 & 571 & 595.7 & 24.7 & D29123 & D32I27 & D314 & 24 & 26 & 2 \\
\hline 2 & 161.2 & -4 & -2 & 2 & 619.2 & 715 & 95.8 & D31/32 & D31/31 & DOI-1 & 27 & 28 & 1 \\
\hline 3 & 159.3 & -36 & -34 & 2 & 570 & 420 & -150 & D26125 & D30I26 & D4l1 & 70 & 52 & -18 \\
\hline 4 & 172.5 & -29 & -5.5 & 23.5 & 720 & 702 & -18 & D35136 & D39136 & D4I0 & 36 & 31 & -5 \\
\hline
\end{tabular}




\section{Discusión}

Esta intervención multicomponente es el primer producto de una investigación que incluye una tercera fase, con un entrenamiento en aceptación y compromiso de 8 semanas más, por lo que los resultados analizados corresponden a la primera intervención con una duración de 5 semanas para dos participantes y de 6 para los otros dos.

Los resultados iniciales de ésta investigación muestran un incremento en el número de pasos y el gasto kilocalórico en todos los participantes, lo que sugiere un camino alentador en el desarrollo del ejercicio físico como hábito saludable, tal como lo encontrado por otros investigadores que han trabajado estrategias conductuales (Donaldson \& Normand, 2009; Valbuena, 2013; Webber, Casey, Mayes, Katsumata \& Mellin, 2016 ).

Los acelerómetros, al igual que los podómetros permiten medir con mayor objetividad la actividad física (Dugdill, Stratton \& Watson, 2009); su uso permitió crear metas tangibles que guiaran semanalmente el comportamiento, mediante un coaching conductual para aumentar el nivel de actividad física en todos los participantes (Valbuena, 2013).

En futuras investigaciones podría ser importante involucrar las mismas estrategias de autocontrol, tal como lo hicieron Webber, Casey, Mayes, Katsumata y Mellin (2016), quienes compararon dos tipos de programas para perder peso en personas con obesidad; uno conductual y otro enfocado en el manejo del estrés; en ambos programas las personas debían realizar 60 minutos de actividad física y una dieta saludable. Sin embargo, el grupo con el programa conductual tenían un entrenamiento en autocontrol para aumentar la actividad física y la reducción de 500 a 1000 kcal de su dieta; este último grupo produjo unas perdidas mayores de peso al final de la intervención.

Esto último podría maximizarse utilizando acelerómetros como los de la presente investigación, los cuales tienen un módulo en la aplicación que permite hacer un cálculo de las calorías consumidas diariamente.
Existió una leve disminución de peso en la mayoría de participantes, pese al poco tiempo de la intervención como ocurrió en el estudio de Gálvez (2017) de tan solo 4 semanas, con una reducción de $54,8 \pm 2,4$ a $52,4 \pm 2,3 \mathrm{~kg}$.

Las pérdidas de peso y de circunferencia de la cintura en personas con sobrepeso y obesidad han sido estudiadas por varios autores; entre ellos Sacks et al. (2009) quienes después de proporcionar cuatro tipos diferentes de dietas en las cuales se modificaban los macronutrientes, no encontraron diferencias significativas con relación a ninguna dieta. En todos los sujetos se presentó una pérdida de peso menor de $0,5 \mathrm{~kg}$ y una disminución del perímetro de cintura de $0,5 \mathrm{~cm}$ durante los primeros 6 meses, respecto a la línea de base. En la presente investigación al combinar actividad física, dieta y coaching conductual, durante el primer mes se obtuvieron unas pérdidas de peso $(0,9$ a $3,3 \mathrm{~kg}$ ) y de perímetro de cintura $(2$ a $5,4 \mathrm{~cm})$ en la mayoría de participantes, en comparación con Sacks et al. (2009).

Respecto a la pérdida de grasa visceral, el participante 1 fue el que disminuyó en éste porcentaje, lo que probablemente se deba a una mayor adherencia al ejercicio cardiorrespiratorio y de fuerza, lo cual se relaciona con mejores puntajes en el test de los 6 minutos y de fuerza prensil. Sánchez, Sánchez y Alfonso (2014), programaron actividad aeróbica combinada con ejercicios de fuerza y flexibilidad, en pacientes con obesidad mórbida, logrando al cabo de 12 semanas "una reducción de la masa grasa total de $2,83 \%$ y de la grasa visceral de $1,43 \%$, mostrando una mejoría no solo de la composición corporal sino además una reducción del riesgo cardiometabólico" (p. 69).

En el caso de los participantes que ganaron porcentaje graso, el participante 3 reportó dificultades con llevar acabo el ejercicio y la dieta, lo cual se evidencia no sólo en los resultados de composición corporal, sino además en los resultados de las pruebas físicas, mientras que el participante 4 reportó problemas de salud.

De acuerdo a Hingorjo, Zehra, Hasan y Qureshi (2017), los estudios han demostrado que los altos niveles de aptitud cardiorrespiratoria en adultos 
se asocian con un menor riesgo de calcificación de las arterias coronarias, de esta manera se contribuye a la prevención de comorbilidades asociadas al sobrepeso y la obesidad como la hipertensión y la diabetes. Es así que los participantes 1 y 2 fueron beneficiados en éste aspecto. Por su parte, el participante 3 no obtuvo mejora en la capacidad cardiorrespiratoria debido a que presentó una adherencia menor al programa de ejercicio frente al resto de participantes, el participante 4 no reflejo mejora debido en parte a una molestia de la rodilla derecha en la semana del test.

De acuerdo a los resultados obtenidos, se logró evidenciar que el entrenamiento concurrente y el aumento de la actividad física en el tiempo libre condujo a cambios positivos en algunos de los componentes en aptitud física. De acuerdo con González (2005), citado por González, Navarro y Pereira (2007), sólo con dos días de entrenamiento físico se pueden conseguir adaptaciones específicas que duran nueve días por medio del entrenamiento con cargas concentradas. Pese a lo anterior, es necesario revisar algunos aspectos que pueden explicar la falta de completa adherencia al programa de entrenamiento concurrente en algunos participantes.

El programa fue suministrado en el escenario deportivo por asistentes, quienes tenían la instrucción de realizar un acompañamiento personalizado las dos primeras semanas con el fin de realizar un adecuado proceso de adaptación. Luego cada participante tenía acceso a su plan y a solucionar dudas sobre el mismo, pero ya no estaría bajo supervisión individual, salvo solicitud expresa, como fue el caso del primer participante. Existe alguna evidencia que sugiere la necesidad de apoyo social por parte del instructor para mantener la práctica de ejercicio (Buckworth y Dishman, 2007).

De otra parte, también es factible revisar de manera constante para futuras investigaciones la intensidad de la actividad física y el esfuerzo percibido, puesto que existe suficiente evidencia a favor de una correlación negativa entre éstas dos variables y el mantenimiento en un programa estructurado (Buckworth \& Dishman, 2007). Es decir, aunque la posibilidad de un entrenamiento estructurado pueda ser inicialmente del gusto de los participantes, es necesario contar con un monitoreo de la adaptación, la percepción y satisfacción con la intensidad establecida en cada sesión.

La retroalimentación semanal se realizó en cuanto al número de pasos y el gasto calórico, más no respecto a la ejecución del plan de entrenamiento. Borg, Kukkonen-Harjula, Fogelholm, y Pasanen (2002) encontraron mayor adherencia a caminar en un grupo de pacientes con sobrepeso que el grupo con entrenamiento de resistencia; sin embargo, cuando ambos grupos carecían de supervisión, el nivel de actividad física disminuía. Esto implica incluir en la retroalimentación la ejecución de las sesiones de entrenamiento concurrente, así como el aumento del automonitoreo para facilitar la adherencia una vez finalice la intervención (Dishman, 1981, citado por Weinberg \& Gould, 2010).

En conclusión, éste programa multidisciplinar con tan solo 6 semanas de intervención presenta progresos en el aumento del gasto calórico y el número de pasos, así como algunos cambios positivos en la composición corporal de los participantes clasificados con obesidad tipo 1 y 2; sin embargo, es necesario fortalecer la adherencia y el seguimiento del entrenamiento concurrente.

\section{Referencias}

American Thoracic Society Statement. (2002). ATS Statement: Guidelines for the six-minute walk test. American Journal of Respiratory and Critical Care Medicine, 166, 111-117. doi: 10.1164/rccm.166/1/111

Ayala, F., Sainz de Baranda, P., de Ste Croix, M., \& Santonja, F. (2012). Fiabilidad y validez de las pruebas sit-and-reach: revisión sistemática. Revista de Andaluza de Medicina del Deporte, 5(2), 57-66.

Bach, P., \& McCracken, S. (s.f.). Best Practice Guidelines for Behavioral Interventions. Recuperado de http://www.bhrm.org/guidelines/ bach-mccraken.pdf 
Barlow, D., Nock, M., \& Hersen, M. (2009). Single Case Experimental Designs. Strategies for Studying Behavior Change. Boston: Pearson.

Block, G., Gillespie, C., Rosenbaum, E., \& Jenson, C. (2000). A rapid food screener to assess fat and fruit and vegetable intake. American Journal of Preventive Medicine. 18(4), 284288.

Borg, P., Kukkonen-Harjula, K., Fogelholm, M., \& Pasanen, M. (2002). Effects of walking or resistance training on weight loss maintenance in obese, middle-aged men: A randomized trial. International Journal of Obesity and Related Metabolic Disorders, 26, 676-683.

Buckworth, J., \& Dishman, R. (2007). Exercise Adherence. En G. Tenenbaum y R. Eklund. Handbook of Sport Psychology (pp.509-536) (3rd ed.). New Jersey: John Wiley \& Sons.

Burgess, E., Hassmén, P., \& Pumpa, K. L. (2017). Determinants of adherence to lifestyle intervention in adults with obesity: a systematic review. Clinical Obesity, 7(3), 123-135. doi: 10.1111/cob.12183

Butland, R., Pang, J., Gross, E., Woodcock, A., \& Geddes, D. (1982). Two, six, and 12 minute walking test in respiratory disease [Abstract]. British Medical Journal, 284 (6329), 16071608.

Cobo-Mejía, E. A., Ochoa, M. E., Ruiz, L.Y., Vargas, D. M., Sáenz, A. M., \& Sandoval Cuellar, C. (2016). Confiabilidad del Senior Fitness Test versión en español, para población adulta mayor en Tunja-Colombia. Archivos De Medicina Del Deporte 33(6), 382-386.

Dietz, W., Baur, L. A., Hall, K., Puhl, R. M., Taveras, E. M., Uauy. R, \& Kopelman, P. (2015). Management of obesity: Improvement of health-care training and systems for prevention and care. Lancet, 385 (9986), 2521-2533. doi: 10.1016/S0140-6736(14)61748-7

Dietz, W., Lee, J., Wechsler, H., Malepati, S., \& Sherry, B. (2007). Health plans' role in pre- venting overweight in children and adolescents. Health Affairs, 26(2), 430-440. doi: 10.1377/hlthaff.26.2.430

Donaldson, J., \& Normand, M. (2009). Using goal setting, self-monitoring and feedback to increase calorie expenditure in obese adults. Behavioral Interventions, 24(2), 73-83. doi: 10.1002/bin.277

Dugdill, L., Stratton, G., \& Watson, P. (2009). Developing the evidence base for physical activity interventions. En L. Dugdill, D. Crone y R. Murphy. Physical Activity and Health Promotion (pp.60-84). Singapore: Wiley-Blackwell.

Ferrari, M. (2013). Estimación de la ingesta por recordatorio 24 horas. Diaeta, 31 (143), 20-25.

Foncillas, A. (7 de mayo de 2016). China engorda sin freno. El periódico. Recuperado de http:// elperiodico.com/

Gálvez, I. (2017). Pérdida de peso y masa grasa con auto-cargas en mujeres. Revista lberoamericana de ciencias de la actividad física y el deporte, 6(2), 30-37.

González, J., Navarro, F., \& Pereira, P. (2007). La planificación del entrenamiento deportivo: cambios vinculados a las nuevas formas de entender las estructuras deportivas contemporáneas. CONEXÕES, 5(1), 1-22. doi: 10.20396/conex.v5i1.8637976

He, Y., Pan, A., Wang, Y., Yang, Y., Xu, J., ... \& Ma, X. (2017). Prevalence of overweight and obesity in 15.8 million men aged $15-49$ years in rural China from 2010 to 2014. Scientific Reports, 7(5012). doi:10.1038/s41598-01704135-4

Hernández, L., \& Blazer, D. (2006). Genetic, Environmental, and Personality Determinants of Health Risk Behaviors. En Autores (Eds.), Genes, Behavior, and the Social Environment: Moving Beyond the Nature/Nurture Debate (pp. 68-89). Washington: National Academies Press. 
Heyward, V. (2008). Evaluación de la Aptitud Física y Prescripción del Ejercicio (5ta ed.). Madrid: Panamericana.

Hingorjo, M. R., Zehra, S., Hasan, Z., \& Qureshi, M. A. (2017).Cardiorespiratory fitness and its association with adiposity indices in young adults. Pakistan Journal of Medical Sciences, 33(3), 659-664. doi: 10.12669/pjms.333.12294

Hoeger, W., \& Hopkins, D. (1992). A comparison of sit and reach and the modified sit and reach in the measurement of flexibility in women. Research Quarterly for Exercise and Sport. 63(2), 191-196. doi: 10.1080/02701367.1992.10607580

Instituto Colombiano de Bienestar Familiar (ICBF). (2010). Encuesta Nacional de la situación nutricional en Colombia 2010, ENSIN. Bogotá: Da Vinci Editores.

Jackson, A., Blair, S., Mahar, M., Wier, L., Ross, R., \& Stuteville, J. (1990). Prediction of functional aerobic capacity without exercise testing. Medicine and Science in Sports and Exercise, 22(6), 863-870.

Jakobsen, L., Rask, I., \& Kondrup, J. (2010). Validation of handgrip strength and endurance as a measure of physical function and quality of life in healthy subjects and patients. Nutrition, 26(5), 542-550. doi: 10.1016/j. nut.2009.06.015

Kang, M., \& Rowe, D. (2015). Issues and challenges in sedentary behavior measurement. Measurement in Physical Education and Exercise Science, 19(3), 105-115. doi: 10.1080/1091367X.2015.1055566

Kazdin, A. (2000). Modificación de Conducta y sus Aplicaciones Prácticas. México: Manual Moderno.

Larsson, U. E., \& Reynisdottir, S. (2008). The sixminute walk test in outpatients with obesity: reproducibility and known group validity. Physiotherapy Research International, 13(2), 84-93. doi:10.1002/pri.398
Millor, N., Lecumberri, P., Gómez, M., MartínezRamírez, A., \& Izquierdo, M. (2013). An evaluation of the 30-s chair stand test in older adults: frailty detection based on kinematic parameters from a single inertial unit. Journal of NeuroEngineering and Rehabilitation, 10(86). doi: 10.1186/1743-0003-10-86

Ministerio de Salud y Protección Social (2016). Análisis de Situación de Salud (ASIS) Colombia, 2016. Recuperado de: https://www. minsalud.gov.co/sites/rid/Lists/BibliotecaDigital/RIDE/VS/ED/PSP/asis-colombia2016.pdf

Ministerio de Salud y Protección Social, Instituto Colombiano de Bienestar Familiar, Instituto Nacional de Salud, Universidad Nacional. (2015). Encuesta Nacional de la Situación Nutricional en Colombia, ENSIN. Recuperado de https://www.minsalud.gov.co/sites/rid/Lists/ Biblioteca Digital/RIDE/VS/ED/PSP/presentacion-lanzamientoensin-2015.pdf

Ministerio de Protección Social (2005). Política Nacional de Prestación de Servicios de Salud. Recuperado de http://www.minsalud.gov. co/Ministerio/Document/ Poli tica\%20Nacional\%20de\%20Prestaci\%C3\%B3n\%20de\%20Servicios\%20de\%20Salud.pdf

Molinero, O., Salguero, A., \& Márquez, S. (2011). Autodeterminación y adherencia al ejercicio: estado de la cuestión. International Journal of Sport Science. 7(25), 287-304. doi: 10.5232/ ricyde2011.02504

Monteiro, P. A., Chen, K. Y., Lira, F. S., Saraiva, B. T. C., Antunes, B. M. M., Campos, E. Z., \& Freitas, I. F. (2015). Concurrent and aerobic exercise training promote similar benefits in body composition and metabolic profiles in obese adolescents. Lipids Health Disease, 14 (153). doi: 10.1186/s12944-015-0152-9

Organización Mundial de la Salud. (febrero de 2017). Actividad física. Recuperado de http:// www.who.int/mediacentre/factsheets/ fs385/es/ 
Peña, G., Heredia, J., Aguilera, J., Da Silva, G., \& Del Rosso, S. (2016). Entrenamiento concurrente de fuerza y resistencia: una revisión narrativa. International Journal of Physical Exercise and Health Science, 1(1). Recuperado de https://g-se.com/entrenamiento-concurrente-de-fuerza-y-resistencia-una-revisionnarrativa-2070-sa-a57cfb27276a24

Ramírez-Vélez, R., Meneses-Echavez, J., GonzálezRuíz, K., \& Correa, J. (2014). Fitness muscular y riesgo cardio-metabólico en adultos jóvenes colombianos. Nutrición Hospitalaria, 30(4), 769-775.

Rikli, R., \& Jones, C. (2001). Senior Fitness Test Manual. Champaign: Human Kinetics.

Sacks, F. M., Bray, G. A., Carey, V. J., Smith, S. R., Ryan, D. H., ... \& Williamson, D. A. (2009). Comparison of weight-loss diets with different compositions of fat, protein and carbohydrates. The New England Journal of Medicine, 360(9), 859-873. doi: 10.1056/NEJMoa0804748

Sánchez, O. L., Sánchez, J. C., \& Alfonso, G. A. (2014). Valoración de un programa de ejercicio físico estructurado en pacientes con obesidad mórbida pendientes de cirugía bariátrica. Nutrición Hospitalaria, 29(1), 64-72.

Segura, E., \& Martínez, F. (2009). Análisis de correlaciones entre los resultados de una prueba de esfuerzo y de la prueba de 6 minutos marcha en población sana. Fisioterapia, 31(6), 241-247. doi: 10.1016/j.ft.2009.05.003
Tudor-Locke, C., Craig, C., Brown, W., Clemes, S., Cocker, K., ... \& Blair, S. (2011). How many steps/day are enough? for adults. International Journal of Behavioral Nutrition and Physical Activity, 8(79), 1-17. doi: 10.1186/1479-58688-79

Valbuena, D. (2013). Evaluating the effectiveness of an internet-based behavioral program for increasing physical activity with and without a behavioral coach (Tesis de Maestría). Recuperado de http://scholarcommons.usf.edu/ etd/4957/

Wadden, T. A., Webb, V. L., Moran, C. H., \& Brooke, A. (2012). Lifestyle Modification for Obesity. New Developments in Diet, Physical Activity and Behavior Therapy, 125(9), 1157-1170. doi: 10.1161/CIRCULATIONAHA.111.039453

Webber, K. H., Casey, E. M., Mayes, L., Katsumata, Y., \& Mellin, L. (2016). A comparison of a behavioral weight loss program to a stress management program: A pilot randomized controlled trial. Nutrition, 32(7-8), 904-909. doi:10.1016/j.nut.2016.01.008

Weinberg, R., \& Gould, D. (2010). Fundamentos de Psicología del Deporte y del Ejercicio Físico. Madrid: Panamericana.

Wells, K., y\& Dillon, E. (1952). The sit and reach - a test of back and leg flexibility. Research Quarterly for Exercise and Sport, 23(1), 115118. doi: 10.1080/10671188.1952.10761965 